\title{
Unusual cause of laryngeal dyspnea and swallowing troubles in a 2-year old child
}

\author{
Monia Attia ${ }^{1,2}$, Imen Bouktif ${ }^{1,2}$, Meriem Affes ${ }^{1,2}$, Sarra Zairi ${ }^{3,4}$, Ikbel Khalfallah ${ }^{2,4}$, Ines Baccouche ${ }^{1,2}$, \\ Henda Neji ${ }^{1,2}$, Saoussen Hantous ${ }^{1,2}$, Khaoula Ben Miled ${ }^{1,2}$ \\ ${ }^{1}$ Department of Radiology, Abderrahmane Mami Hospital, Ariana; ${ }^{2}$ Faculty of Medicine of Tunis, University of Tunis \\ El Manar; ${ }^{3}$ Department of Thoracic and Cardiovascular Surgery, Abderrahmane Mami Hospital, Ariana; ${ }^{4}$ Department \\ of Pulmonology, Abderrahmane Mami Hospital, Ariana, Tunisia
}

\begin{abstract}
Plexiform neurofibromas are rare benign tumors developed from peripheral nervous system often associated with neurofibromatosis type 1 . We report the case of multifocal plexiform neurofibromas in a 2-year-old child with cervical mass obstructing the trachea causing respiratory distress. A cervical ultrasound examination was performed followed by enhanced CT and MRI. Imaging revealed an expansive cervical mass extended from the base of the skull to the mediastinum associated with similar pelvic and sacral foraminal masses. The target like MRI aspect on T2-weighted images was suggestive of the neural origin. Biopsy under ultrasound control confirmed the diagnosis of plexiform neurofibroma.
\end{abstract}

\section{Introduction}

Neurofibromas are rare benign tumors developed from peripheral nervous system often associated with neurofi-

Correspondence: Monia Attia, Department of Radiology,

Abderrahmane Mami Hospital, Rue de l'Hôpital, 2080 Ariana, Tunisia.

E-mail: attiamonia82@gmail.com

Key words: Neurofibromatosis; child; dyspnoea.

Conflict of interest: All authors declare no conflict of interest.

Availability of data and materials: All data underlying the findings are fully available.

Consent for publication: The manuscript does not contain any individual person's data in any form.

Received for publication: 2 February 2020.

Accepted for publication: 2 July 2020.

${ }^{\circ}$ Copyright: the Author(s), 2020

Licensee PAGEPress, Italy

Monaldi Archives for Chest Disease 2020; 90:1244

doi: 10.4081/monaldi.2020.1244

This article is distributed under the terms of the Creative Commons Attribution Noncommercial License (by-nc 4.0) which permits any noncommercial use, distribution, and reproduction in any medium, provided the original author(s) and source are credited. bromatosis type 1 or Von Recklinghausen disease. Imaging techniques including computed tomography (CT) and magnetic resonance imaging (MRI) are helpful to suggest the diagnosis and to specify tumor extension.

We report the case of multifocal plexiform neurofibromas involving the neck, the mediastinum and the pelvic in a 2-year-old child with a respiratory distress.

\section{Case Report}

A 2-year-old boy was referred to our institution for evaluation and management of a left side neck mass with respiratory distress. Symptoms were progressing for more than 3 months. Respiratory symptoms were prominent and mistaken for recurrent episodes of bronchiolitis. They mainly consisted of dyspnea and few aspiration episodes. Physical examination revealed a firm irregular painless mass in the left hemi-neck with normal overlying skin. There were no palpable lymph nodes otherwise. The child had polypnea and the pulse oximeter revealed normal oxygen saturation.

Multiple cutaneous café-au-lait spots were noticed on his back ranging from 5 to $10 \mathrm{~mm}$ in diameter with smooth borders. No neurological disorder was found. Routine laboratory tests were normal. Antenatal monitoring was without particularity and no family history of hereditary disease was known. A cervical ultrasound examination showed an infiltrating solid mass, with lobulated margins surrounding the common carotid artery and internal jugular vein. Enlarged lymph nodes were suspected. Contrast-enhanced CT examination was performed from skull basis to pubic symphysis (Figure 1). It revealed multiple infiltrating cervical masses extending to mediastinum, compressing the trachea and the whole upper aerodigestive tract. Besides, ill-defined masses were noted in the presacral region associated with spherical lesions enlarging sacral foramina. These lesions were hypodense with minimal enhancement after contrast injection.

Magnetic resonance imaging (MRI) was indicated to better characterize and evaluate the tumor's extension. It showed ill-defined lesions developed in neck deep spaces, extending to the infra-temporal fossa, infiltrating the parotid gland and enlarging the left jugular foramen. Thorax exploration revealed mediastinal masses in continuity with cervi- 
cal lesions. In pelvic region, there were multiple infiltrating masses surrounding sacral plexus and well-defined masses enlarging sacral foramina. The signal was hypointense on T1- weighted images. Target-like aspect was noticed on T2weighted images with central low-signal intensity and peripheral high-signal intensity suggesting neural origin (Figure 2).

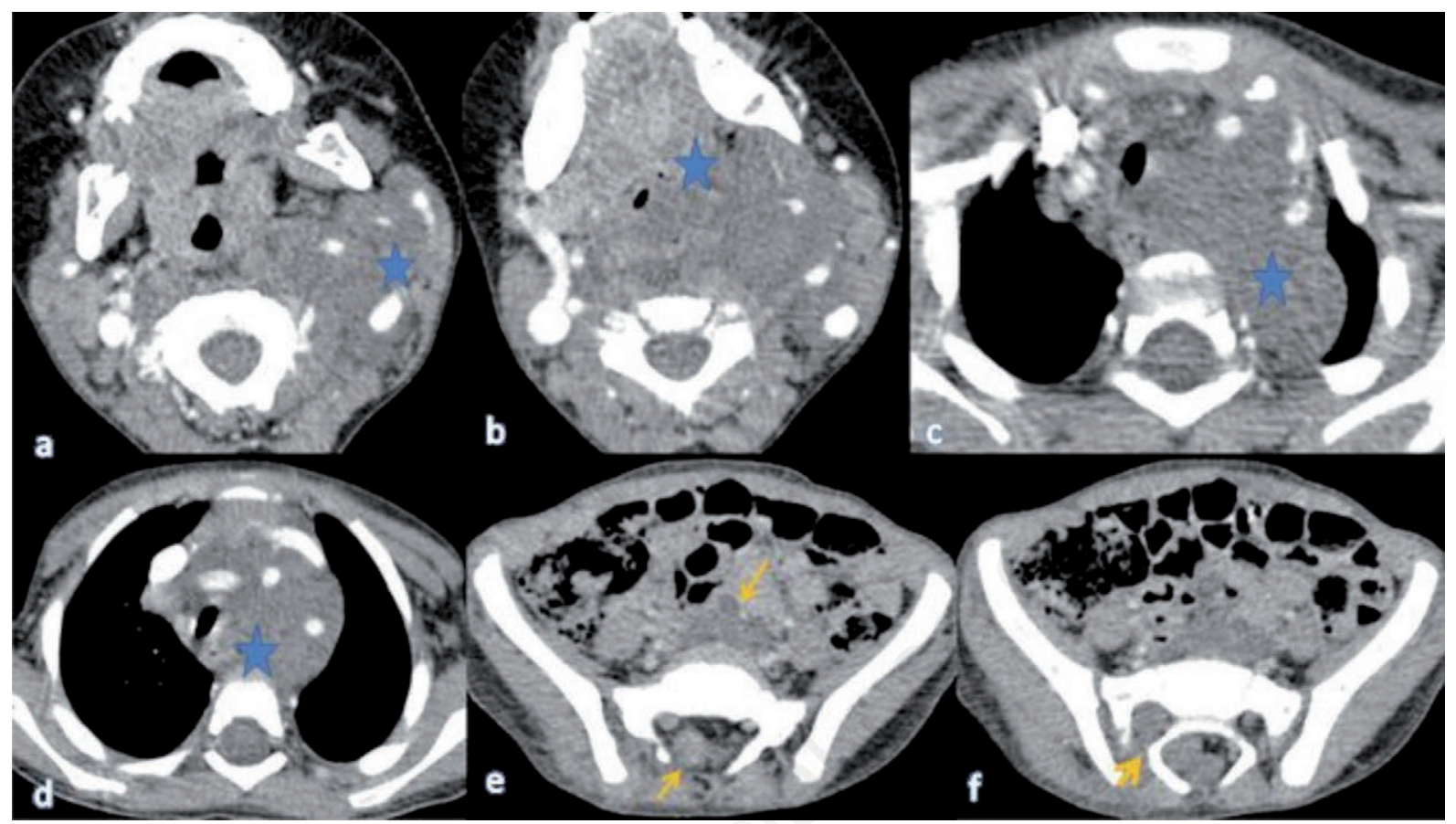

Figure 1. Contrast-enhanced CT scan. a,b) Axial sections demonstrating hypodense solid masses of left side of the neck and skull base compressing the oropharyngeal pathway (asterisk). b,c) Axial sections of thoracic level showing infiltrative mediastinal masses surrounding the vessels and compressing the trachea (asterisk). d,e) Axial sections through the pelvic areas demonstrating ill-defined tum ors enlarging sacral foramina (arrow).

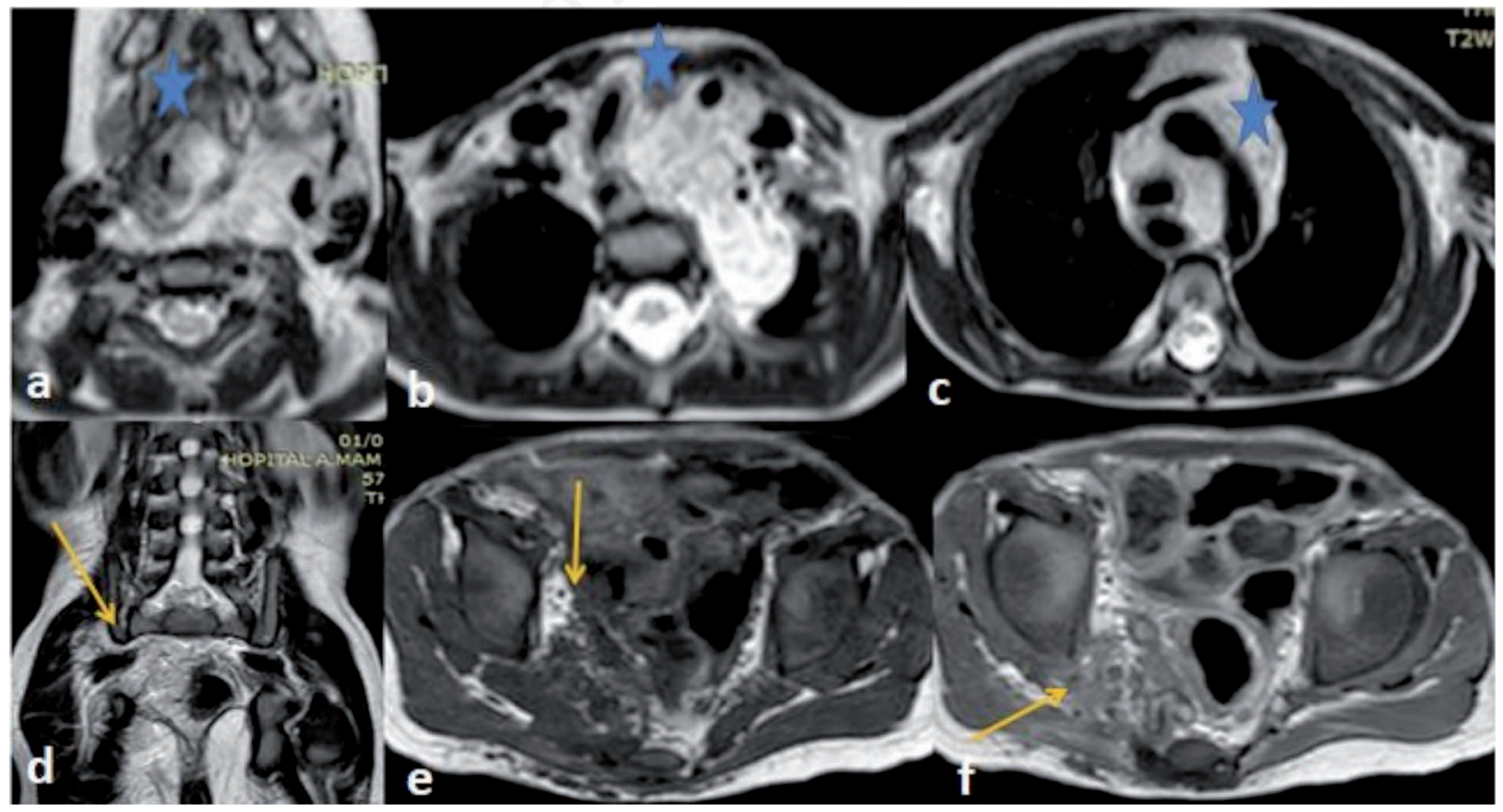

Figure 2. Axial T2 sequences (a,b,c) ill-defined lesions developed in neck deep spaces in continuity with mediastinal mass compressing the oropharyngeal pathway and trachea (asterisk). Coronal T2 sequence (d); axial T1 before and after contrast injection (e,f) of the pelvic region: multiple infiltrating masses surrounding sacral plexus and well-defined rounded masses enlarging sacral foramina with $\mathrm{T} 2$ target sign (arrow). 
Neurofibromatosis was suspected and percutaneous biopsy of the cervical mass was performed under general anesthesia and ultrasound control. Anatomo-pathological examination confirmed the diagnosis of neurofibroma. Genetic testing was positive for NF1 (Figure 3).

\section{Discussion}

Neurofibromatosis describes a group of genetic disorders that primarily affect the cell growth of neural tissues. At least eight forms of neurofibromatosis have been known [1]; the most common form is neurofibromatosis (nf) type 1 (von Recklinghausen). It occurs in 1 of 4000 births, generally inherited in an autosomal dominant pattern with variable penetrance. However, some cases may result from spontaneous mutation [2]. There is no sex predilection [1].

A defect in a tumor suppressor gene on chromosome 17 leads to a high risk for developing a variety of benign and malignant tumors [3]. Neurofibromas and optic gliomas are the most common tumors found in NF-1 [2].

Histologically, neurofibroma are a proliferation of thick wavy collagen bundles with varying degrees of myxoid degeneration [4]. Three subtypes of neurofibromas exist : cutaneous, subcutaneous and plexiform which is specific for the disease [1]. Plexiform neurofibromas are typically congenital and mainly diagnosed between 2 and 5 years [5]. They can involve any peripheral nerve and are generally extensive with undefined margins [1]. They occur frequently in the head, neck, face, and larynx , especially the cranial and upper cervical nerves $[1,2]$. In the reported case, the main mass was localized in cervical deep spaces. The cranial nerves commonly involved are the fifth, ninth, and tenth. These tumors can develop in the posterior mediastinum with schwannoma as main differential diagnosis.

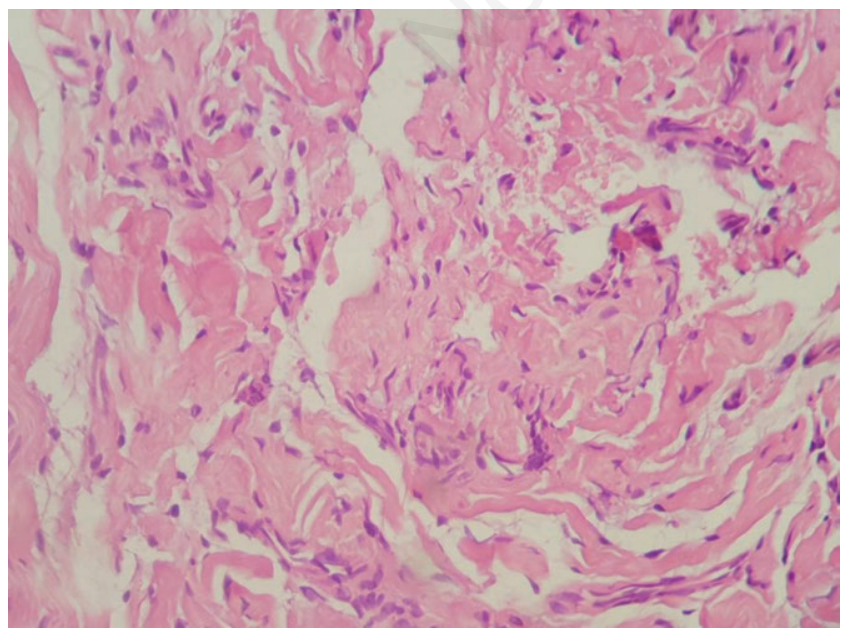

Figure 3. Proliferation consisting of spindle-shaped cells with small, undulating nuclei and scanty cytoplasm. The matrix is rich in collagen keychains. H\&E, magnification: 200x.
They originate from the intercostal or sympathetic nerve, and occasionally from the vagus nerve [5].

The diagnosis should be done because of the risk of malignant transformation described in up to $15 \%$ of NF1 patients [6]. Although frequent, plexiform neurofibromas are not pathognomonic of NF-1 [7]. They represent one of the seven criteria of the clinical diagnosis of NF1 established by the $1988 \mathrm{NIH}$ Consensus Development Conference $[8,9]$. Plexiform neurofibroma's natural history varies and some tumors may remain asymptomatic especially when they are superficial [5]. They also may progress into large and invasive lesions causing disfigure and hemi facial hypertrophy as seen in our case [10]. Noticed symptoms range from minor discomfort to extreme pain [1]. Clinical presentations depend on the prevalent location. Ocular motility disturbance, neurological deficits are most common symptoms [11]. Dyspnea and respiratory failure, as reported in this case, are due to upper airway compression.

MRI and computed tomography are two imaging modalities performed when neurofibromas are suspected. They both provide the site and the expansion of the tumors [5]. On CT, plexiform neurofibroma appears as a hypodense mass and has a variable degree of enhancement [12]. The aspect is usually of a non-specific infiltrative lesion [13]. MRI is the gold standard imaging technique for the diagnosis and investigation of neurofibromas by providing useful and precise data about the location, the assessment, and the extension of the tumor. Neurofibroma appears hypointense on T1-weighted images with a mild enhancement after contrast injection [13]. A target-like sign seen on T2-weighted images is typical of neurofibromas, especially in deep plexiform neurofibromas because of the tumor architecture with central nerve fibers and peripheral myxoid matrix. This sign consists of a central hypointense zone surrounded by a high signal on T2- weighted sequence. However, and particularly on superficial locations, the absence of a target-like appearance does not rule out the diagnosis [1]. In our case, the target like sign was found on T2 weighted MR images suggesting the diagnosis.

Neurofibromas are usually diagnosed clinically. anatomopathological examination is mandatory to exclude malignant transformation [11]. The treatment is generally surgical with resection of deforming masses and cancerous lesions when they occur. However, $20 \%$ of recidivism cases are usually reported despite a suitable approach $[12,13]$.

\section{Conclusions}

Plexiform neurofibromas are an uncommon cause of dyspnea and wheezing in a 2-year-old child, especially without any family history of neurofibromatosis. Clinical examination and magnetic resonance imaging are usually sufficient to make the diagnosis. Radical treatment of multifocal lesions is challenging especially when surgery is not possible. 


\section{References}

1. Anand R, Yadav DS, Yadav V, et al. Plexiform neurofibroma in a 16-year-old girl. Radiol Case Rep 2012;7:708.

2. Wise JB, Cryer JE, Belasco JB, et al. Management of head and neck plexiform neurofibromas in pediatric patients with neurofibromatosis type 1. Arch Otolaryngol Neck Surg 2005;131:712-8.

3. Greinwald J, Derkay CS, Schechter GL. Management of massive head and neck neurofibromas in children. Am J Otolaryngol 1996;17:136-42.

4. Hassell DS, Bancroft LW, Kransdorf MJ, et al. Imaging appearance of diffuse neurofibroma. Am J Roentgenol 2008;190:582-8.

5. Kolker S, Wunder JS, Roche-Nagle G. Hybrid resection of a giant thigh plexiform neurofibroma. Int J Surg Case Rep 2015;8:1-4.

6. Fadda MT, Verdino G, Mustazza MC, et al. Intra-parotid facial nerve multiple plexiform neurofibroma in patient with NF1. Int J Pediatr Otorhinolaryngol 2008;72:553-7.
7. Lin V, Daniel S, Forte V. Is a plexiform neurofibroma pathognomonic of neurofibromatosis type I? Laryngoscope 2004; 114:1410-4.

8. Carter RL. Tumors of the head and neck: clinical and pathological considerations. J R Soc Med 1982;75:57.

9. No authors listed. Neurofibromatosis. Conference statement. National Institutes of Health Consensus Development Conference. Arch Neurol 1988;45:575-8.

10. McGaughran JM, Harris DI, Donnai D, et al. A clinical study of type 1 neurofibromatosis in north west England. J Med Genet 1999;36:197-203.

11. Cihangiroglu M, Yilmaz S, Topsakal C, et al. Laryngeal neurofibroma associated with neurofibromatosis type 2. Am J Neuroradiol 2002;23:1637-9.

12. Plotkin SR, Davis SD, Robertson KA, et al. Sleep and pulmonary outcomes for clinical trials of airway plexiform neurofibromas in NF1. Neurology 2016;87:S13-20.

13. Needle MN, Cnaan A, Dattilo J, et al. Prognostic signs in the surgical management of plexiform neurofibroma: The Children's Hospital of Philadelphia experience, 1974-1994. J Pediatr 1997;131:678-82. 